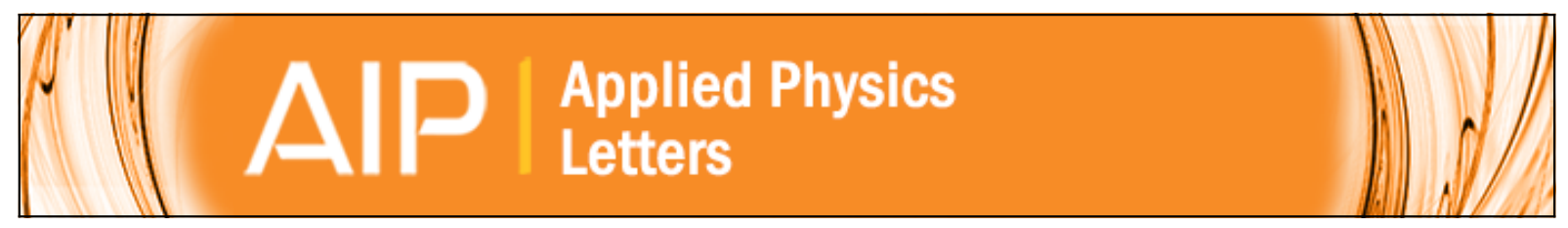

\title{
Efficient excitation transfer from polymer to nanocrystals
}

Tung-Wah Frederick Chang, Sergei Musikhin, Ludmila Bakueva, Larissa Levina, Margaret A. Hines, Paul W. Cyr , and Edward H. Sargent

Citation: Applied Physics Letters 84, 4295 (2004); doi: 10.1063/1.1755414

View online: http://dx.doi.org/10.1063/1.1755414

View Table of Contents: http://scitation.aip.org/content/aip/journal/apl/84/21?ver=pdfcov

Published by the AIP Publishing

\section{Articles you may be interested in}

Stark shifts and exciton dissociation in CdSe nanoparticle grafted conjugated polymer

Appl. Phys. Lett. 100, 151908 (2012); 10.1063/1.3701719

Efficient energy transfer in layered hybrid organic/inorganic nanocomposites: A dual function of semiconductor nanocrystals

Appl. Phys. Lett. 96, 083109 (2010); 10.1063/1.3319838

Direct observation of the size dependence of Dexter energy transfer from polymer to small $\mathrm{PbS}$ quantum dots Appl. Phys. Lett. 93, 083106 (2008); 10.1063/1.2975847

White electroluminescence from a hybrid polymer-GaN:Mg nanocrystals device Appl. Phys. Lett. 91, 093132 (2007); 10.1063/1.2772667

Size-tunable infrared (1000-1600 nm) electroluminescence from PbS quantum-dot nanocrystals in a semiconducting polymer

Appl. Phys. Lett. 82, 2895 (2003); 10.1063/1.1570940

\section{AIP $\mid$ APL Photonics \\ APL Photonics is pleased to announce Benjamin Eggleton as its Editor-in-Chief}

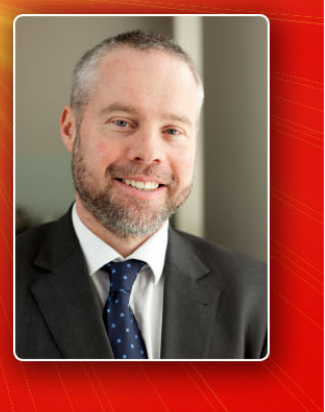




\title{
Efficient excitation transfer from polymer to nanocrystals
}

\author{
Tung-Wah Frederick Chang, ${ }^{\text {a) }}$ Sergei Musikhin, Ludmila Bakueva, Larissa Levina, \\ Margaret A. Hines, Paul W. Cyr, and Edward H. Sargent \\ Department of Electrical and Computer Engineering, University of Toronto, 10 King's College Road, \\ Toronto, M5S-3G4, Canada
}

(Received 2 September 2003; accepted 27 March 2004; published online 7 May 2004)

\begin{abstract}
We quantify experimentally the efficiency of excitation transfer from a semiconducting polymer matrix to quantum dot nanocrystals. We study $5 \pm 0.5 \mathrm{~nm} \mathrm{PbS}$ nanocrystals embedded in MEH-PPV (poly[2-methoxy-5-(2'-ethylhexyloxy-p-phenylenevinylene)]) polymer. We determine the excitation transfer efficiency from normalized photoluminescence excitation measurements. When the composites are made using as-synthesized $\mathrm{PbS}$ nanocrystals capped by oleate ligands, the excitation transfer efficiency is about $20 \%$. Replacing these ligands with shorter chains results in a factor-of-3 enhancement in the excitation transfer efficiency. Our findings provide guidance to the realization of efficient electroluminescent devices. (C) 2004 American Institute of Physics.
\end{abstract}

[DOI: $10.1063 / 1.1755414]$

Quantum dots confine excitons and offer the size-tunable optoelectronic properties of artificial atoms. Colloidal nanocrystal (NC) quantum dots, ${ }^{1,2}$ being solution-processible, have been used as the light-emitting material in polymerbased light-emitting devices (LED) in the visible ${ }^{3}$ and infrared ${ }^{4-6}$ range. In optoelectronics, size tunability permits spectral control of absorption for photovoltaic, ${ }^{7}$ stimulated emission, ${ }^{8}$ and electroluminescence ${ }^{4-6}$ applications.

The organometallically synthesized NCs are capped by a layer of organic ligands (Fig. 1) to prevent aggregation and thereby preserve their quantum-confined energy levels. These ligands are hydrocarbon chains terminated by a functional group. They have much larger energy gaps than the NC material and have been demonstrated to inhibit charge transfer. ${ }^{9}$ Since excitation transfer from polymer to NCs is a necessary step for electrically pumped radiation from NCs, this capping layer is thought to be a major hurdle to achieving high electroluminescence quantum efficiencies in polymer-NC composites. The best efficiencies are typically in the $1 \%$ range, whereas inorganic LEDs and lasers have external quantum efficiencies in the 10\%-60\% range.

Mechanisms for this excitation transfer include the Förster and Dexter mechanisms. While the Förster mechanism is a dipole-dipole coupling process and Dexter an electron transport process, both have strong though different dependencies on the distance separating the donor and the acceptor involved. To determine the contribution of the excitation transfer process to the overall electroluminescence efficiency, it is important to quantify the efficiency of the transfer process.

We used PbS NCs synthesized as in Refs. 6 and 10. The $\mathrm{NCs}$ have an average diameter of $5 \mathrm{~nm}$ and a luminescence peak at $1500 \mathrm{~nm}$. Their size tunability over the photoluminescence peak range 1000-1600 $\mathrm{nm}$ has previously been demonstrated.

We employed four types of capping ligands: the 18-

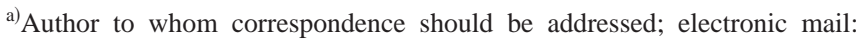
fred.chang@utoronto.ca
}

carbon-single-chain oleate terminated by a carboxyl group (herein labeled C18), the 18-carbon-single-chain octadecylamine terminated by an amine group (A18), the 12-carbon dodecylamine (A12), and the 8-carbon octylamine (A8). Composite solutions were made by thoroughly mixing NCin-toluene and MEH-PPV-in-toluene solutions of known concentration. The composite films (named C18-P, A18-P, A12-P, and A8-P) were made by dropping each of the composite solutions on a cleaned quartz substrate and allowing it to dry. Pure NC films (named C18-N, A18-N, A12-N, and A8-N) were made by the same method, with the absence of MEH-PPV.

We investigated the efficiency of net transfer of excitations in the polymer to the NCs using the normalized photoluminescence excitation spectrum $Q(\lambda)$ of NCs. $Q(\lambda)$ is, for each excitation wavelength $\lambda$, the rate of photon emission by the NCs normalized to the rate of direct optical absorption by the NCs of the incident light at that excitation wavelength $\lambda$. It is obtained from

$$
Q(\lambda) \propto \frac{E_{n}(\lambda)}{\left(1-T_{c}(\lambda)\right) F_{n T}(\lambda)},
$$

where $E_{n}(\lambda)$ is the measured photoluminescence excitation spectrum of NCs for the photoluminescence peak at 1500 $\mathrm{nm}, T_{c}(\lambda)$ is the measured transmittance spectrum of the

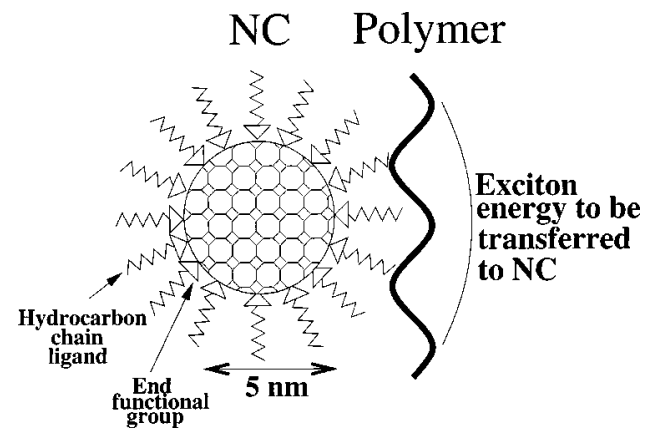

FIG. 1. PbS NC capped by ligands consists of a hydrocarbon chain and an end functional group. Excitation may be transferred from polymer to NC. 


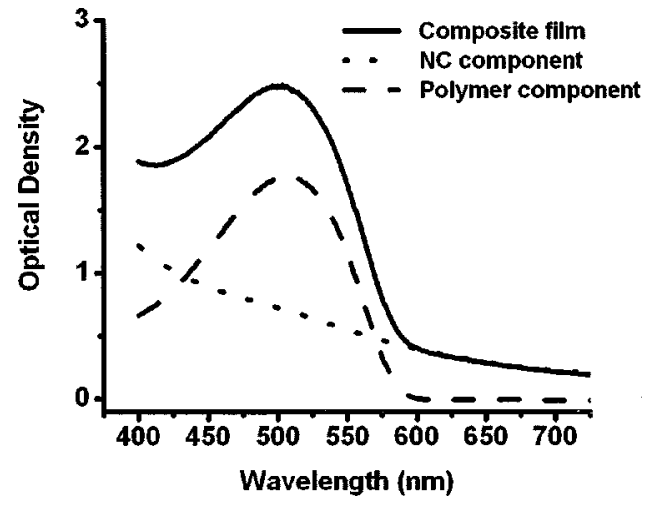

FIG. 2. Absorption spectra of a composite film (solid line) and its deconvolved MEH-PPV polymer (dashed line) and NC (dotted line) components.

composite film, and $F_{n T}(\lambda)$ is the fraction of the absorbed incident excitation light absorbed by the NC part of the composite film. This fraction is obtained from

$$
F_{n T}(\lambda)=\frac{A_{n}(\lambda)}{A_{c}(\lambda)},
$$

where $A_{c}(\lambda)$ and $A_{n}(\lambda)$ are the measured optical density spectra of the composite film and its deconvolved NC component (Fig. 2), respectively. The absorption spectra were measured using a Cary 500 UV-Vis-NIR spectrophotometer. The photoluminescence excitation spectra were measured using a PTI spectrofluorometer with a tungsten-halogen lamp.

The absorption spectra in Fig. 2 show that only the NC component of the composite film can be excited for excitation wavelengths longer than $620 \mathrm{~nm}$. MEH-PPV has negligible absorption in this range. At excitation wavelengths shorter than $620 \mathrm{~nm}, Q(\lambda)$, plotted in Fig. 3, of the composite films for emission at $1500 \mathrm{~nm}$ rises abruptly as the polymer begins absorbing incident light and transferring excitations to the NCs, resulting in enhanced infrared luminescence from the NCs. The extent of the increase in $Q$ with this onset of polymer absorption allows us to quantify the transfer efficiency from polymer to NCs. Pure NC films show featureless $Q(\lambda)$ spectra and confirm that the onset of polymer absorption is responsible for the peak in $Q(\lambda)$ of the composite films centered at $500 \mathrm{~nm}$. [Note that in Fig. 3,

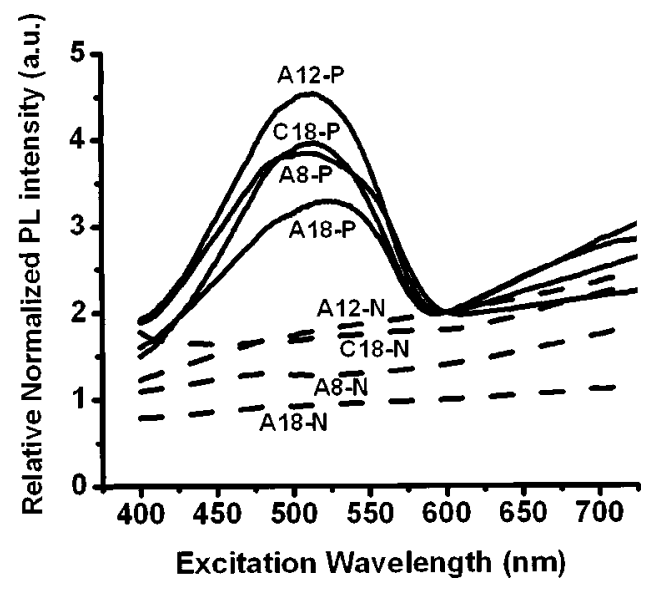

FIG. 3. Relative normalized photoluminescence excitation spectra $Q(\lambda)$ of NCs for composite films C18-P, A18-P, A12-P, and A8-P (solid lines) and NC-only films C18-N, A18-N, A12-N, and A8-N (dashed lines) for the four kinds of ligands.

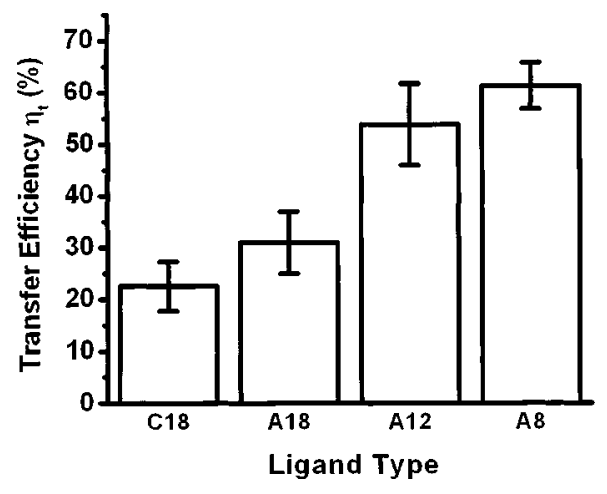

FIG. 4. The absolute transfer efficiency $\eta_{t}$ of excitations from MEH-PPV to NCs for the four ligand choices: oleate (C18), octadecylamine (A18), dodecylamine (A12), and octylamine (A8).

the vertical axis is in arbitrary units; curves have been scaled to facilitate comparison since it is the relative spectral shape of $Q(\lambda)$ that reveals excitation transfer.]

Phenomenologically, the rate of photon emission $P_{n}$ by the NCs at a given excitation wavelength is

$$
P_{n}=k\left(L_{n}+L_{p} \eta_{t}\right),
$$

where $L_{n}$ and $L_{p}$ are the rates of photon absorption by NC and polymer, $\eta_{t}$ is the efficiency with which excitations generated in the polymer are transferred to the NCs, and $k$ is a constant of proportionality. $Q$ is given by

$$
Q=\frac{P_{n}}{L_{n}}=k\left(1+\frac{L_{p}}{L_{n}} \eta_{t}\right) .
$$

Since the ratio of $L_{p}$ to $L_{n}$ can be obtained from the ratio of the polymer to the NC components of the deconvolved optical density spectra of the composite film (Fig. 3), $\eta_{t}$ can be extracted from the $Q(\lambda)$ curves using Eq. (4).

The efficiency $\eta_{t}$ is plotted in Fig. 4. Three main conclusions can be drawn. First, the role of the end functional group in this excitation transfer process plays a modest role: $\eta_{t}$ of the C18 and A18 ligands are similar. Second, the transfer efficiency increases as the ligand chain length decreases. An improvement by a factor of almost 3 is achieved by exchanging the as-synthesized oleate ligand having a $2 \mathrm{~nm} 18$ carbon chain for shorter ligands having $1.5 \mathrm{~nm} \mathrm{12-carbon,} \mathrm{or}$ $1 \mathrm{~nm}$ 8-carbon chains. Third, in all cases the transfer efficiency is in the tens of percent.

Previously, two distinct phenomena-internal quantum efficiency of NCs in polymers and transfer efficiency across the ligand layer-have been held responsible for the low electroluminescence quantum efficiency of devices. ${ }^{4,6}$ Our results suggest that even relatively long insulating ligands do not severely impede the transfer of excitations from polymers to NCs, although shorter chains can improve the transfer efficiency. This suggests that considerable freedom may be exploited in engineering ligands and ligand-NC interfaces for high internal quantum efficiency.

The authors thank the support of Nortel Networks, the Natural Sciences and Engineering Research Council of Canada under the Collaborative Research and Development Program, the Materials and Manufacturing Ontario, the Canada Research Chairs Program, the Canada Foundation for Innovation, and the Ontario Innovation Trust. 
${ }^{1}$ A. P. Alivisatos, Science 271, 933 (1996).

${ }^{2}$ M. Shim and P. Guyot-Sionnest, Nature (London) 407, 981 (2000).

${ }^{3}$ S. Coe, W.-K. Woo, M. Bawendi, and V. Bulović, Nature (London) 420, 800 (2002).

${ }^{4}$ N. Tessler, V. Medvedev, M. Kazes, S. Kan, and U. Banin, Science 295, 1506 (2002).

${ }^{5}$ J. S. Steckel, S. Coe-Sullivan, V. Bulović, and M. Bawendi, Adv. Mater. (Weinheim, Ger.) 15, 1862 (2003).

${ }^{6}$ L. Bakueva, S. Musikhin, M. A. Hines, T.-W. F. Chang, M. Tzolov,
G. D. Scholes, and E. H. Sargent, Appl. Phys. Lett. 82, 2895 (2003).

${ }^{7}$ W. U. Huynh, J. J. Dittmer, and A. P. Alivisatos, Science 295, 2425 (2002).

${ }^{8}$ V. I. Klimov, A. A. Mikhailovsky, S. Xu, A. Malko, J. A. Hollingsworth, C. A. Leatherdale, H.-J. Eisler, and M. G. Bawendi, Science 290, 314 (2000).

${ }^{9}$ M. Drndić, M. V. Jarosz, N. Y. Morgan, M. A. Kastner, and M. G. Bawendi, Science 300, 1280 (2003).

${ }^{10}$ M. A. Hines and G. D. Scholes, Adv. Mater. (Weinheim, Ger.) 15, 1844 (2003). 